\title{
Some inequalities for warped product pseudo-slant submanifolds of nearly Kenmotsu manifolds
}

\author{
Akram Ali ${ }^{* *} \mathbb{D}$, Wan Ainun Mior Othman ${ }^{1}$ and Cenap Ozel
}

\section{"Correspondence:}

akramali133@gmail.com

${ }^{1}$ Institute of Mathematical Sciences,

Faculty of Science, University of

Malaya, Kuala Lumpur, 50603,

Malaysia

Full list of author information is

available at the end of the article

\begin{abstract}
In this paper, we study non-trivial warped product pseudo-slant submanifolds of nearly Kenmotsu manifolds. In the beginning, we obtain some lemmas and then develop the general sharp inequalities for mixed totally geodesic warped products pseudo-slant submanifolds. The equality cases are also considered.
\end{abstract}

MSC: 53C40; 53C42; 53B25

Keywords: inequalities; mean curvature; pseudo-slant; warped product; warping functions; nearly Kenmotsu; mixed totally geodesic

\section{Introduction}

The geometric inequalities of warped product submanifolds have been studied actively since Chen [1] introduced the notion of a CR-warped product submanifold in a Kaehler manifold and obtained inequalities for the second fundamental form in terms of warping functions. In fact, different types of warped product submanifolds of different structures are studied for the last 14 years (see [2]). Recently, Sahin [3] established a general inequality for warped product pseudo-slant (also named hemi-slant) isometrically immersed in a Kaehler manifold for mixed totally geodesic. Later on, Uddin, et al. [4-6] obtained some inequalities of warped product submanifolds in different structures. In the present paper, we extend this idea into a nearly Kenmotsu manifold and derive the geometric inequalities of non-trivial warped product pseudo-slant submanifolds which are the natural extensions of CR-warped product submanifolds. Every CR-warped product submanifold is a nontrivial warped product pseudo-slant submanifold of the forms $M_{\perp} \times_{f} M_{\theta}$ and $M_{\theta} \times_{f} M_{\perp}$ with slant angle $\theta=0$. First of all we consider non-trivial warped product pseudo-slant submanifolds of the form $M=M_{\perp} \times_{f} M_{\theta}$ and $M_{\theta} \times_{f} M_{\perp}$ such that $M_{\theta}$ and $M_{\perp}$ are properslant and anti-invariant submanifolds. Next we establish inequalities involving the second fundamental form, slant angle, and warping functions.

The paper is organized as follows: In Section 2, we review some preliminary formulas, definitions and address the study of pseudo-slant submanifolds of nearly Kenmotsu manifolds. In Section 3, we study warped product pseudo-slant submanifolds of a nearly Kenmotsu manifold and obtain some lemmas. In Section 4, we define an orthonormal frame

(c) 2015 Ali et al. This article is distributed under the terms of the Creative Commons Attribution 4.0 International License (http://creativecommons.org/licenses/by/4.0/), which permits unrestricted use, distribution, and reproduction in any medium, provided you give appropriate credit to the original author(s) and the source, provide a link to the Creative Commons license, and indicate if changes were made. 
for warped product pseudo-slant submanifolds and then obtain general sharp inequalities for the second fundamental form in terms of warping functions and slant immersions.

\section{Preliminaries}

Let $\widetilde{M}$ be a $(2 m+1)$-dimensional almost contact manifold with almost contact structure $(\varphi, \xi, \eta)$ where $\varphi$ is a $(1,1)$ tensor field, $\xi$ is the structure vector field and $\eta$ is a dual 1 -form satisfying the following property:

$$
\varphi^{2}=-I+\eta \otimes \xi, \quad \eta(\xi)=1, \quad \varphi(\xi)=0, \quad \eta \circ \varphi=0 .
$$

On an almost contact manifold there exists a Riemannian metric $g$ which satisfies the following:

$$
g(\varphi U, \varphi V)=g(U, V)-\eta(U) \eta(V), \quad \eta(U)=g(U, \xi)
$$

for any $U, V$ tangent to $\widetilde{M}$. Then an almost contact manifold $\widetilde{M}$ equipped with a Riemannian metric $g$ is called an almost contact metric manifold $(\widetilde{M}, g)$. Furthermore, an almost contact metric manifold is known to be a Kenmotsu manifold [7] if

$$
\left(\widetilde{\nabla}_{U} \varphi\right) V=g(\varphi U, V) \xi-\eta(V) \varphi U
$$

and

$$
\widetilde{\nabla}_{U \xi}=U-\eta(U) \xi
$$

for any vector fields $U, V$ on $\widetilde{M}$, where $\widetilde{\nabla}$ denote the Riemannian connection with respect to $g$. Then an almost contact metric manifold $\widetilde{M}$ is said to be a nearly Kenmotsu manifolds [7], if

$$
\left(\widetilde{\nabla}_{U} \varphi\right) V+\left(\widetilde{\nabla}_{V} \varphi\right) U=-\eta(U) \varphi V-\eta(V) \varphi U
$$

for any $U, V$ tangent to $\widetilde{M}$. We shall use the symbol $\Gamma(T \widetilde{M})$ to denote the Lie algebras of vector fields on a manifold $\widetilde{M}$.

Let $M$ be a Riemannian manifold that is isometrically immersed in an almost contact metric manifold $\widetilde{M}$ and denote by the same symbol $g$ the Riemannian metric induced on $M$. Let $\Gamma(T M)$ and $\Gamma\left(T^{\perp} M\right)$ be the Lie algebras of vector fields tangent to $M$ and normal to $M$, respectively, and $\nabla^{\perp}$ the induced connection on $T^{\perp} M$. Denote by $\mathcal{F}(M)$ the algebra of smooth functions on $M$ and by $\Gamma(T M)$ the $\mathcal{F}(M)$-module of smooth sections of $T M$ over $M$. Denote by $\nabla$ the Levi-Civita connection of $M$ then the Gauss and Weingarten formulas are given by

$$
\begin{gathered}
\widetilde{\nabla}_{U} V=\nabla_{U} V+h(U, V), \\
\widetilde{\nabla}_{U} N=-A_{N} U+\nabla_{U}^{\perp} N,
\end{gathered}
$$

for each $U, V \in \Gamma(T M)$ and $N \in \Gamma\left(T^{\perp} M\right)$, where $h$ and $A_{N}$ are the second fundamental form and the shape operator (corresponding to the normal vector field $N$ ), respectively, 
for the immersion of $M$ into $\tilde{M}$. They are related as

$$
g(h(U, V), N)=g\left(A_{N} U, V\right) .
$$

Now for any $U \in \Gamma(T M)$, we write

$$
\varphi U=P U+F U,
$$

where $P U$ and $F U$ are the tangential and normal components of $\varphi U$, respectively. Similarly for any $N \in \Gamma\left(T^{\perp} M\right)$, we have

$$
\varphi N=t N+f N
$$

where $t N$ (resp. $f N$ ) are the tangential (resp. normal) components of $\varphi N$. A submanifold $M$ is said to be totally geodesic and totally umbilical, if $h(U, V)=0$ and $h(U, V)=g(U, V) H$, respectively.

There is another class of submanifolds, which is called the class of slant submanifold. For each non-zero vector $U$ tangent to $M$ at $p$, such that $U$ is not proportional to $\xi_{p}$, we denote by $0 \leq \theta(U) \leq \pi / 2$, the angle between $\varphi U$ and $T_{p} M$, which is called the Wirtinger angle. If the angle $\theta(U)$ is constant for all $U \in T_{p} M-\langle\xi(p)\rangle$ and $p \in M$, then $M$ is called a slant submanifold [8] and the angle $\theta$ is called the slant angle of $M$. Obviously if $\theta=0$, $M$ is invariant and if $\theta=\pi / 2, M$ is anti-invariant submanifold. A slant submanifold is said to be proper slant if it is neither invariant nor anti-invariant.

In an almost contact metric manifold, in fact, Cabrerizo et al. [9] obtained the following theorem.

Theorem 2.1 Let $M$ be a submanifold of an almost contact metric manifold $\tilde{M}$ such that $\xi \in T M$. Then $M$ is slant if and only if there exists a constant $\lambda \in[0,1]$ such that

$$
P^{2}=\lambda(-I+\eta \otimes \xi)
$$

Furthermore, in such a case, $\theta$ is the slant angle and it satisfies $\lambda=\cos ^{2} \theta$.

Hence, for a slant submanifold $M$ of an almost contact metric manifold $\widetilde{M}$, we have the following relations which are consequences of Theorem 2.1:

$$
\begin{aligned}
& g(P U, P V)=\cos ^{2} \theta\{g(U, V)-\eta(U) \eta(V)\}, \\
& g(F U, F V)=\sin ^{2} \theta\{g(U, V)-\eta(U) \eta(V)\},
\end{aligned}
$$

for any $U, V \in \Gamma(T M)$.

Moreover, we define pseudo-slant submanifolds of almost contact manifolds by using the slant distribution given in [10]. However, pseudo-slant submanifolds were defined by Carriazo [11] under the name anti-slant submanifolds as a particular class of bi-slant submanifolds. The definition of pseudo-slant is as follows.

Definition 2.1 A submanifold $M$ of an almost contact manifold $\widetilde{M}$ is said to be pseudoslant submanifold, if there exist two orthogonal distributions $\mathcal{D}^{\perp}$ and $\mathcal{D}^{\theta}$ such that: 
(i) $T M=\mathcal{D}^{\theta} \oplus \mathcal{D}^{\perp} \oplus\langle\xi\rangle$, where $\langle\xi\rangle$ is a 1-dimensional distribution spanned by $\xi$.

(ii) $\mathcal{D}^{\perp}$ is an anti-invariant distribution under $\varphi$ i.e., $\varphi \mathcal{D}^{\perp} \subseteq T^{\perp} M$.

(iii) $\mathcal{D}^{\theta}$ is a slant distribution with slant angle $\theta \neq 0, \frac{\pi}{2}$.

Let $m_{1}$ and $m_{2}$ be the dimensions of the distributions $\mathcal{D}^{\perp}$ and $\mathcal{D}^{\theta}$, respectively. If $m_{2}=0$, then $M$ is an anti-invariant submanifold. If $m_{1}=0$ and $\theta=0$, then $M$ is an invariant submanifold. If $m_{1}=0$ and $\theta \neq 0, \frac{\pi}{2}$, then $M$ is a proper-slant submanifold, or if $\theta=\frac{\pi}{2}$, then $M$ is an anti-invariant submanifold and if $\theta=0$, then $M$ is a semi-invariant submanifold. Since $\mu$ is an invariant subspace of a normal bundle $T^{\perp} M$, then in the case of a pseudo-slant submanifold, the normal bundle $T^{\perp} M$ can be decomposed as follows:

$$
T^{\perp} M=\varphi \mathcal{D}^{\perp} \oplus F \mathcal{D}^{\theta} \oplus \mu,
$$

where $\mu$ is an even dimensional invariant subbundle of $T^{\perp} M$. A pseudo-slant submanifold is said to be mixed totally geodesic if $h(X, Z)=0$, for all $X \in \Gamma\left(\mathcal{D}^{\perp}\right)$ and $Z \in \Gamma\left(\mathcal{D}^{\theta}\right)$. Now let $\left\{e_{1}, e_{2}, \ldots, e_{n}\right\}$ be an orthonormal basis of the tangent space $T M$ and $e_{r}$ belong to the orthonormal basis $\left\{e_{n+1}, e_{n+2}, \ldots, e_{m}\right\}$ of a normal bundle $T^{\perp} M$, then we define

$$
h_{i j}^{r}=g\left(h\left(e_{i}, e_{j}\right), e_{r}\right) \quad \text { and } \quad\|h\|^{2}=\sum_{i, j=1}^{n} g\left(h\left(e_{i}, e_{j}\right), h\left(e_{i}, e_{j}\right)\right) .
$$

As a consequence, for a differentiable function $\lambda$ on $M$, we have

$$
\|\nabla \lambda\|^{2}=\sum_{i=1}^{n}\left(e_{i}(\lambda)\right)^{2}
$$

where the gradient $\operatorname{grad} \lambda$ is defined by $g(\nabla \lambda, X)=X \lambda$, for any $X \in \Gamma(T M)$.

\section{Warped product submanifolds}

In [12], Bishop and O'Neill, defined the notion of warped product manifolds to construct examples of Riemannian manifolds with a negative curvature. These manifolds are natural generalizations of Riemannian product manifolds. They defined these manifolds as follows: Let $\left(M_{1}, g_{1}\right)$ and $\left(M_{2}, g_{2}\right)$ be two Riemannian manifolds and $f: M_{1} \rightarrow(0, \infty)$ a positive differentiable function on $M_{1}$. Consider the product manifold $M_{1} \times M_{2}$ with its canonical projections $\pi_{1}: M_{1} \times M_{2} \rightarrow M_{1}, \pi_{2}: M_{1} \times M_{2} \rightarrow M_{2}$ and the projection maps given by $\pi_{1}(p, q)=p$ and $\pi_{2}(p, q)=q$ for every $t=(p, q) \in M_{1} \times M_{2}$. The warped product $M=M_{1} \times_{f} M_{2}$ is the product manifold $M_{1} \times M_{2}$ equipped with the Riemannian structure such that

$$
\|U\|^{2}=\left\|\pi_{1} *(U)\right\|^{2}+f^{2}\left(\pi_{1}(p)\right)\left\|\pi_{2} *(U)\right\|^{2}
$$

for any tangent vector $U \in \Gamma\left(T_{t} M\right)$, where $*$ is the symbol of the tangent maps. Thus we have $g=g_{1}+f^{2} g_{2}$. The function $f$ is called the warping function on $M$. It was defined in $[12]$ and we have the following.

Lemma 3.1 [12] Let $M=M_{1} \times_{f} M_{2}$ be warped product manifolds. For any $X, Y \in \Gamma\left(T M_{1}\right)$ and $Z, W \in \Gamma\left(T M_{2}\right)$ : 
(i) $\nabla_{X} Y \in \Gamma\left(T M_{1}\right)$,

(ii) $\nabla_{Z} X=\nabla_{X} Z=(X \ln f) Z$,

(iii) $\nabla_{Z} W=\nabla_{Z}^{\prime} W-g(Z, W) \nabla \ln f$,

where $\nabla$ and $\nabla^{\prime}$ denote the Levi-Civita connections on $M$ and $M_{2}$, respectively. On the other hand, $\nabla \ln f$, the gradient of $\ln f$, is defined as $g(\nabla \ln f, U)=U \ln f$. A warped product manifold $M=M_{1} \times_{f} M_{2}$ is said to be trivial if the warping function $f$ is constant. If $M=$ $M_{1} \times_{f} M_{2}$ is a warped product manifold then $M_{1}$ is a totally geodesic and $M_{2}$ is a totally umbilical submanifold of $M$. First of all we give some preparatory lemmas.

Lemma 3.2 Let $M=M_{\perp} \times_{f} M_{\theta}$ be a warped product pseudo-slant submanifold of a nearly Kenmotsu manifold $\tilde{M}$ such that the structure vector field $\xi$ is tangent to $M_{\perp}$. Then

$$
g(h(X, P X), \varphi Z)=g(h(X, Z), F P X)+\frac{1}{3}\{\eta(Z)-(Z \ln f)\} \cos ^{2} \theta\|X\|^{2},
$$

for any $X \in \Gamma\left(T M_{\theta}\right)$ and $Z \in \Gamma\left(T M_{\perp}\right)$.

Proof Let $M=M_{\perp} \times_{f} M_{\theta}$ be a warped product pseudo-slant submanifold of a nearly Kenmotsu manifold $\tilde{M}$, then by (2.6), we have

$$
g(h(X, P X), \varphi Z)=-g\left(\varphi \widetilde{\nabla}_{X} P X, Z\right)
$$

Thus using the covariant derivatives of $\varphi$, we obtain

$$
g(h(X, P X), \varphi Z)=g\left(\left(\widetilde{\nabla}_{X} \varphi\right) P X, Z\right)-g\left(\widetilde{\nabla}_{X} \varphi P X, Z\right) .
$$

From the nearly Kenmotsu structure (2.5) and Theorem 2.1, we derive

$$
g(h(X, P X), \varphi Z)=-g\left(\left(\widetilde{\nabla}_{P X} \varphi\right) X, Z\right)-\cos ^{2} \theta g\left(\widetilde{\nabla}_{X} Z, X\right)+g(h(X, Z), F P X) .
$$

Thus from Lemma 3.1(ii) and the covariant derivative of an endomorphism $\varphi$, we obtain

$$
\begin{aligned}
g(h(X, P X), \varphi Z)= & -g\left(\widetilde{\nabla}_{P X} \varphi X, Z\right)-g\left(\widetilde{\nabla}_{P X} X, \varphi Z\right) \\
& +g(h(X, Z), F P X)-\cos ^{2} \theta(Z \ln f)\|X\|^{2} .
\end{aligned}
$$

Using (2.9) and (2.7), we arrive at

$$
\begin{aligned}
2 g(h(X, P X), \varphi Z)= & g\left(\nabla_{P X} Z, P X\right)-g\left(\widetilde{\nabla}_{P X} F X, Z\right) \\
& +g(h(X, Z), F P X)-\cos ^{2} \theta(Z \ln f)\|X\|^{2} .
\end{aligned}
$$

Finally, using Lemma 3.1(ii) and (2.12), we get

$$
2 g(h(X, P X), \varphi Z)=g(h(P X, Z), F X))+g(h(X, Z), F P X) .
$$

Again for any $X \in \Gamma\left(T M_{\theta}\right)$ and $Z \in \Gamma\left(T M_{\perp}\right)$, we have

$$
g(h(X, Z), F P X)=g\left(\widetilde{\nabla}_{Z} X, F P X\right)
$$


From the fact that $\xi$ is tangent to $M_{\perp},(2.6)$, and (2.9), we obtain

$$
g(h(X, Z), F P X)=-g\left(\varphi \widetilde{\nabla}_{Z} X, P X\right)+\cos ^{2} \theta g\left(\nabla_{Z} X, X\right) .
$$

Then from the definition of the covariant derivative of $\varphi$ and Lemma 3.1(ii), we can derive

$$
g(h(X, Z), F P X)=g\left(\left(\widetilde{\nabla}_{Z} \varphi\right) X, P X\right)-g\left(\widetilde{\nabla}_{Z} \phi X, P X\right)+\cos ^{2} \theta(Z \ln f)\|X\|^{2} .
$$

Thus by using the structure equation (2.5) and (2.5) and (2.12), the above equation takes the form

$$
\begin{aligned}
g(h(X, Z), F P X)= & -g\left(\left(\widetilde{\nabla}_{X} \varphi\right) Z, P X\right)-\eta(Z) \cos ^{2} \theta\|X\|^{2}-g\left(\nabla_{Z} P X, P X\right) \\
& -g\left(\widetilde{\nabla}_{Z} F X, P X\right)+\cos ^{2} \theta(X \ln f)\|Z\|^{2} .
\end{aligned}
$$

Using Lemma 3.1(ii), (2.7), and (2.12), we arrive at

$$
\begin{aligned}
g(h(X, Z), F P X)= & -g\left(\widetilde{\nabla}_{X} \varphi Z, P X\right)-g\left(\widetilde{\nabla}_{X} Z, \varphi P X\right) \\
& -\eta(Z) \cos ^{2} \theta\|X\|^{2}+g(h(Z, P X), F X) .
\end{aligned}
$$

Equation (2.7) and Theorem 2.1 for a slant submanifold give us

$$
\begin{aligned}
g(h(X, Z), F P X)= & g\left(A_{\varphi Z} X, P X\right)+\cos ^{2} \theta g\left(\nabla_{X} Z, X\right)-\eta(Z) \cos ^{2} \theta\|X\|^{2} \\
& -g\left(\widetilde{\nabla}_{X} Z, F P X\right)+g(h(Z, P X), F X) .
\end{aligned}
$$

Finally, from Lemma 3.1(ii) and (2.6), we derive

$$
\begin{aligned}
g(h(X, P X), \varphi Z)= & 2 g(h(X, Z), F P X)-\{(Z \ln f)-\eta(Z)\} \cos ^{2} \theta\|X\|^{2} \\
& -g(h(Z, P X), F X) .
\end{aligned}
$$

Thus from (3.1) and (3.2), we get

$$
g(h(X, P X), \varphi Z)-g(h(X, Z), F P X)=\frac{1}{3}\{\eta(Z)-(Z \ln f)\} \cos ^{2} \theta\|X\|^{2},
$$

which is our final result. This completes the proof of the lemma.

Lemma 3.3 Let $M=M_{\perp} \times_{f} M_{\theta}$ be a warped product pseudo-slant submanifold of a nearly Kenmotsu manifold $\tilde{M}$. Then:

(iii) $g(h(X, X), \varphi Z)=g(h(Z, X), F X)$,

(iv) $g(h(P X, P X), \varphi Z)=g(h(Z, P X), F P X)$,

for any $X \in \Gamma\left(T M_{\theta}\right)$ and $Z \in \Gamma\left(T M_{\perp}\right)$.

Proof For $X \in \Gamma\left(T M_{\theta}\right)$ and $Z \in \Gamma\left(T M_{\perp}\right)$, we have

$$
g(h(X, X), \varphi Z)=g\left(\widetilde{\nabla}_{X} X, \varphi Z\right)=-g\left(\varphi \widetilde{\nabla}_{X} X, Z\right) .
$$


From the definition of the covariant derivative of a tensor field $\varphi$, we get

$$
\left.g(h(X, X), \varphi Z)=g\left(\widetilde{\nabla}_{X} \varphi\right) X, Z\right)-g\left(\widetilde{\nabla}_{X} \phi X, Z\right) .
$$

Then from (2.5) and (2.2), we obtain

$$
g(h(X, X), \varphi Z)=g\left(\widetilde{\nabla}_{X} Z, P X\right)-g\left(\widetilde{\nabla}_{X} F X, Z\right) .
$$

Thus from Lemma 3.1(ii) and (2.6), the above equation can be written as

$$
g(h(X, X), \varphi Z)=(Z \ln f) g(X, P X)+g\left(A_{F X} X, Z\right) .
$$

Since $X$ and $P X$ are orthogonal vector fields and considering (2.8), we arrive at

$$
g(h(X, X), \varphi Z)=g(h(X, Z), F X)
$$

which is the first result of the lemma. Interchanging $X$ by $P X$ in (3.3), we get the last result of the lemma. This completes the proof of the lemma.

Lemma 3.4 Assume we have a $M=M_{\theta} \times_{f} M_{\perp}$ non-trivial warped product pseudo-slant submanifold of a nearly Kenmotsu manifold $\tilde{M}$. Then:

(i) $g(h(Z, Z), F P X)=g(h(Z, P X), \varphi Z)+\{\eta(X)-(X \ln f)\} \cos ^{2} \theta\|Z\|^{2}$,

(ii) $g(h(Z, Z), F X)=g(h(Z, X), \varphi Z)-(P X \ln f)\|Z\|^{2}$,

for any $X \in \Gamma\left(T M_{\theta}\right)$ and $Z \in \Gamma\left(T M_{\perp}\right)$, where the structure vector field $\xi$ is tangent to $M_{\theta}$.

Proof From (2.6), and (2.9), we get

$$
g(h(Z, Z), F P X)=g\left(\widetilde{\nabla}_{Z} Z, F P X\right)=g\left(\widetilde{\nabla}_{Z} Z, \varphi P X\right)-g\left(\widetilde{\nabla}_{Z} Z, P^{2} X\right) .
$$

From Theorem 2.1, we obtain

$$
g(h(Z, Z), F P X)=-g\left(\varphi \widetilde{\nabla}_{Z} Z, P X\right)+\cos ^{2} \theta\left\{g\left(\widetilde{\nabla}_{Z} Z, X\right)-\eta(X) g\left(\widetilde{\nabla}_{Z} Z, \xi\right)\right\} .
$$

Using the property of a Riemannian connection and the covariant derivative of an endomorphism, we derive

$$
\begin{aligned}
g(h(Z, Z), F P X)= & g\left(\left(\widetilde{\nabla}_{Z} \varphi\right) Z, P X\right)-g\left(\widetilde{\nabla}_{Z} \phi Z, P X\right)-\cos ^{2} \theta g\left(\nabla_{Z} X, Z\right) \\
& +\cos ^{2} \theta \eta(X) g\left(\widetilde{\nabla}_{Z} \xi, Z\right) .
\end{aligned}
$$

Thus from the fact that in a nearly Kenmotsu manifolds $(\xi \ln f)=1$ [13] and from Lemma 3.1(ii), we arrive at

$$
g(h(Z, Z), F P X)=g(h(Z, P X), \phi Z)+\{\eta(X)-(X \ln f)\} \cos ^{2} \theta\|Z\|^{2},
$$

which is the first part of the lemma. The second part of the lemma can easily be found by interchanging $X$ by $P X$ in the above equation. This completes the proof of the lemma. 


\section{Inequalities of warped product submanifolds}

\subsection{Inequality for a warped product pseudo-slant submanifold of the form $M_{\perp} \times_{f} M_{\theta}$}

In this section, we obtain a geometric inequality of warped product pseudo-slant submanifold in terms of the second fundamental form such that $\xi$ is tangent to the antiinvariant submanifold and the mixed totally geodesic submanifold. First of all we define an orthonormal frame for later use.

Let $M=M_{\perp} \times_{f} M_{\theta}$ be a $m$-dimensional warped product pseudo-slant submanifold of a $2 n+1$-dimensional nearly Kenmotsu manifold $\widetilde{M}$ with $M_{\theta}$ of dimension $d_{1}=2 \beta$ and $M_{\perp}$ of dimension $d_{2}=\alpha+1$, where $M_{\theta}$ and $M_{\perp}$ are the integral manifolds of $\mathcal{D}^{\theta}$ and $\mathcal{D}^{\perp}$, respectively. Then we consider that $\left\{e_{1}, e_{2}, \ldots, e_{\alpha}, e_{d_{2}=\alpha+1}=\xi\right\}$ and $\left\{e_{\alpha+2}=\right.$ $\left.e_{1}^{*}, \ldots, e_{\alpha+\beta+1}=e_{\beta}^{*}, e_{\alpha+\beta+2}=e_{\beta+1}^{*}=\sec \theta P e_{1}^{*}, \ldots, e_{\alpha+1+2 \beta}=e_{2 \beta}^{*}=\sec \theta P e_{\beta}^{*}\right\}$ are orthonormal frames of $\mathcal{D}^{\perp}$ and $\mathcal{D}^{\theta}$, respectively. Thus the orthonormal frames of the normal subbundles $\varphi \mathcal{D}^{\perp}, F \mathcal{D}^{\theta}$, and $\mu$, respectively, are $\left\{e_{m+1}=\bar{e}_{1}=\varphi e_{1}, \ldots, e_{m+\alpha}=\bar{e}_{\alpha}=\varphi e_{\alpha}\right\}$, $\left\{e_{m+\alpha+1}=\bar{e}_{\alpha+1}=\tilde{e}_{1}=\csc \theta F e_{1}^{*}, \ldots, e_{m+\alpha+\beta}=\bar{e}_{\alpha+\beta}=\tilde{e}_{\beta}=\csc \theta F e_{\beta}^{*}, e_{m+\alpha+\beta+1}=\bar{e}_{\alpha+\beta+1}=\tilde{e}_{\beta+1}=\right.$ $\left.\csc \theta \sec \theta F P e_{1}^{*}, \ldots, e_{m+\alpha+2 \beta}=\bar{e}_{\alpha+2 \beta}=\tilde{e}_{2 \beta}=\csc \theta \sec \theta F P e_{\beta}^{*}\right\}$, and $\left\{e_{2 m-1}=\bar{e}_{m}, \ldots, e_{2 n+1}=\right.$ $\left.\bar{e}_{2(n-m+1)}\right\}$.

Theorem 4.1 Let $M=M_{\perp} \times_{f} M_{\theta}$ be a m-dimensional mixed totally geodesic warped product pseudo-slant submanifold of a $2 n+1$-dimensional nearly Kenmotsu manifold $\widetilde{M}$ such that $\xi \in \Gamma\left(T M_{\perp}\right)$, where $M_{\perp}$ is an anti-invariant submanifold of dimension $d_{2}$ and $M_{\theta}$ is a proper-slant submanifold of dimension $d_{1}$ of $\widetilde{M}$. Then:

(i) The squared norm of the second fundamental form of $M$ is given by

$$
\|h\|^{2} \geq \frac{2 \beta}{9} \cos ^{2} \theta\left\{\left\|\nabla^{\perp} \ln f\right\|^{2}-1\right\} .
$$

(ii) The equality holds in (4.1), if $M_{\perp}$ is totally geodesic and $M_{\theta}$ is a totally umbilical submanifold into $\widetilde{M}$.

Proof The squared norm of the second fundamental form is defined as

$$
\|h\|^{2}=\left\|h\left(\mathcal{D}^{\theta}, \mathcal{D}^{\theta}\right)\right\|^{2}+\left\|h\left(\mathcal{D}^{\perp}, \mathcal{D}^{\perp}\right)\right\|^{2}+2\left\|h\left(\mathcal{D}^{\theta}, \mathcal{D}^{\perp}\right)\right\|^{2}
$$

Since $M$ is mixed totally geodesic,

$$
\|h\|^{2}=\left\|h\left(\mathcal{D}^{\perp}, \mathcal{D}^{\perp}\right)\right\|^{2}+\left\|h\left(\mathcal{D}^{\theta}, \mathcal{D}^{\theta}\right)\right\|^{2} .
$$

Then with (2.15), we obtain

$$
\|h\|^{2} \geq \sum_{l=m+1}^{2 n+1} \sum_{i, j=1}^{2 \beta} g\left(h\left(e_{i}^{*}, e_{j}^{*}\right), e_{l}\right)^{2} .
$$


Since the above equation can be expressed as in the components of $\varphi \mathcal{D}^{\perp}, F \mathcal{D}^{\theta}$, and $v$, we derive

$$
\begin{aligned}
\|h\|^{2} \geq & \sum_{l=1}^{\alpha} \sum_{i, j=1}^{2 \beta} g\left(h\left(e_{i}^{*}, e_{j}^{*}\right), \bar{e}_{l}\right)^{2}+\sum_{l=\alpha+1}^{2 \beta+\alpha} \sum_{i, j=1}^{2 \beta} g\left(h\left(e_{i}^{*}, e_{j}^{*}\right), \bar{e}_{l}\right)^{2} \\
& +\sum_{l=m}^{2(n-m+1)} \sum_{i, j=1}^{2 \beta} g\left(h\left(e_{i}^{*}, e_{j}^{*}\right), \bar{e}_{l}\right)^{2} .
\end{aligned}
$$

Leaving all the terms except the first, we get

$$
\|h\|^{2} \geq \sum_{l=1}^{\alpha} \sum_{i, j=1}^{2 \beta} g\left(h\left(e_{i}^{*}, e_{j}^{*}\right), \bar{e}_{l}\right)^{2} .
$$

Using another adapted frame for $\mathcal{D}^{\theta}$, we derive

$$
\begin{aligned}
\|h\|^{2} \geq & \sum_{i=1}^{\alpha} \sum_{r, k=1}^{\beta} g\left(h\left(e_{r}^{*}, e_{k}^{*}\right), \bar{e}_{i}\right)^{2}+\sec ^{2} \theta \sum_{i=1}^{\alpha} \sum_{r, k=1}^{\beta} g\left(h\left(P e_{r}^{*}, e_{k}^{*}\right), \bar{e}_{i}\right)^{2} \\
& +\sec ^{2} \sum_{i=1}^{\alpha} \sum_{r, k=1}^{\beta} g\left(h\left(e_{r}^{*}, P e_{k}^{*}\right), \bar{e}_{i}\right)^{2}+\sec ^{4} \theta \sum_{i=1}^{p} \sum_{r, k=1}^{\beta} g\left(h\left(P e_{r}^{*}, P e_{k}^{*}\right), \bar{e}_{i}\right)^{2} .
\end{aligned}
$$

Then for a mixed totally geodesic submanifold, the first and last terms of the right hand side in the above equation vanish identically by using Lemma 3.3, and we obtain

$$
\|h\|^{2} \geq 2 \sec ^{2} \theta \sum_{i=1}^{\alpha} \sum_{r=1}^{\beta} g\left(h\left(P e_{r}^{*}, e_{r}^{*}\right), \bar{e}_{i}\right)^{2} .
$$

Thus from Lemma 3.2, for a mixed totally geodesic submanifold and using the fact that $\eta\left(e_{i}\right)=0, i=1,2, \ldots, d_{2}-1$ for an orthonormal frame, we arrive at

$$
\|h\|^{2} \geq \frac{2}{9} \cos ^{2} \theta \sum_{i=1}^{\alpha} \sum_{r=1}^{\beta}\left(\bar{e}_{i} \ln f\right)^{2} g\left(e_{r}^{*}, e_{r}^{*}\right)^{2} .
$$

Now we add and subtract the same term $\xi \ln f$ in (4.4), getting

$$
\|h\|^{2} \geq \frac{2}{9} \cos ^{2} \theta \sum_{i=1}^{\alpha+1} \sum_{r=1}^{\beta}\left(\bar{e}_{j} \ln f\right)^{2} g\left(e_{r}^{*}, e_{r}^{*}\right)^{2}-\frac{2}{9} \cos ^{2} \theta \sum_{r=1}^{\beta}(\xi \ln f)^{2} g\left(e_{r}^{*}, e_{r}^{*}\right)^{2} .
$$

It well known that $\xi \ln f=1$ [13] for a warped product submanifold of a nearly Kenmotsu manifold. Thus the above equation gives

$$
\|h\|^{2} \geq \frac{2 \beta}{9} \cos ^{2} \theta\left\{\left\|\nabla^{\perp} \ln f\right\|^{2}-1\right\} .
$$

If the equality holds above, then from the terms left in (4.2), we obtain the following condition from the first term:

$$
h\left(\mathcal{D}^{\perp}, \mathcal{D}^{\perp}\right)=0,
$$


which means that $M_{\perp}$ is totally geodesic in $\widetilde{M}$. Similarly, from the second and third terms in (4.3), we derive

$$
g\left(h\left(\mathcal{D}^{\theta}, \mathcal{D}^{\theta}\right), F \mathcal{D}^{\theta}\right)=0, \quad g\left(h\left(\mathcal{D}^{\theta}, \mathcal{D}^{\theta}\right), v\right)=0,
$$

which implies that

$$
h\left(\mathcal{D}^{\theta}, \mathcal{D}^{\theta}\right) \perp F \mathcal{D}^{\theta}, \quad h\left(\mathcal{D}^{\theta}, \mathcal{D}^{\theta}\right) \perp v \quad \Longrightarrow \quad h\left(\mathcal{D}^{\theta}, \mathcal{D}^{\theta}\right) \subset \phi \mathcal{D}^{\perp} .
$$

We have Lemma 3.2, which shows that $M_{\theta}$ is a totally umbilical into $\widetilde{M}$ due to being totally umbilical in $M$. So equality holds. This completes the proof of the theorem.

\subsection{Inequality for a warped product pseudo-slant submanifold of the form}

$M_{\theta} \mathrm{x}_{f} M_{\perp}$

In this section, we obtain an inequality of warped product pseudo-slant subamnifolds such that the structure vector field $\xi$ is tangent to the slant submanifold $M_{\theta}$. Taking $\xi$ tangent to $\mathcal{D}^{\theta}$, then we use the last frame.

Theorem 4.2 Let $M=M_{\theta} \times_{f} M_{\perp}$ be a m-dimensional mixed totally geodesic warped product pseudo-slant submanifold of a $2 n+1$-dimensional nearly Kenmotsu manifold $\widetilde{M}$ such that $\xi \in \Gamma\left(T M_{\theta}\right)$, where $M_{\perp}$ is an anti-invariant submanifold of dimension $d_{2}=\alpha$ and $M_{\theta}$ is a proper-slant submanifold of dimension $d_{1}=2 \beta+1$ of $\tilde{M}$. Then:

(i) The squared norm of the second fundamental form of $M$ is given by

$$
\|h\|^{2} \geq \alpha\left\{\csc ^{2} \theta\left(\left\|\nabla^{\theta} \ln f\right\|^{2}-1\right)-\sum_{i=1}^{\beta}\left(e_{i}^{*} \ln f\right)^{2}\right\} .
$$

(ii) If equality holds identically in (4.5), then $M_{\theta}$ is a totally geodesic submanifold and $M_{\perp}$ is a totally umbilical submanifold of $\widetilde{M}$, respectively.

Proof We start by the definition of the second fundamental form

$$
\|h\|^{2}=\left\|h\left(\mathcal{D}^{\theta}, \mathcal{D}^{\theta}\right)\right\|^{2}+\left\|h\left(\mathcal{D}^{\perp}, \mathcal{D}^{\perp}\right)\right\|^{2}+2\left\|h\left(\mathcal{D}^{\theta}, \mathcal{D}^{\perp}\right)\right\|^{2} .
$$

Since $M$ is a mixed totally geodesic, we get

$$
\|h\|^{2}=\left\|h\left(\mathcal{D}^{\perp}, \mathcal{D}^{\perp}\right)\right\|^{2}+\left\|h\left(\mathcal{D}^{\theta}, \mathcal{D}^{\theta}\right)\right\|^{2} .
$$

Thus by (2.15), we obtain

$$
\|h\|^{2} \geq \sum_{l=m+1}^{2 n+1} \sum_{r, k=1}^{\alpha} g\left(h\left(e_{r}, e_{k}\right), e_{l}\right)^{2} .
$$

The above equation can be expressed in the components of $\phi \mathcal{D}^{\perp}, F \mathcal{D}^{\theta}$, and $v$ as

$$
\begin{aligned}
\|h\|^{2} \geq & \sum_{l, r, k=1}^{\alpha} g\left(h\left(e_{r}, e_{k}\right), \bar{e}_{l}\right)^{2}+\sum_{l=\alpha+1}^{2 \beta+\alpha} \sum_{r, k=1}^{\alpha} g\left(h\left(e_{r}, e_{k}\right), \bar{e}_{l}\right)^{2} \\
& +\sum_{l=m}^{2(n-m+1)} \sum_{r, k=1}^{\alpha} g\left(h\left(e_{r}, e_{k}\right), \bar{e}_{l}\right)^{2} .
\end{aligned}
$$


We shall leave all the terms, except the second term, and we get

$$
\|h\|^{2} \geq \sum_{l=1}^{2 \beta} \sum_{r, k=1}^{\alpha} g\left(h\left(e_{r}, e_{k}\right), \tilde{e}_{l}\right)^{2}
$$

Thus using the adapted frame for $F \mathcal{D}^{\theta}$, we derive

$$
\|h\|^{2} \geq \csc ^{2} \theta \sum_{j=1}^{\beta} \sum_{r=1}^{\alpha} g\left(h\left(e_{r}, e_{r}\right), F e_{j}^{*}\right)^{2}+\csc ^{2} \theta \sec ^{2} \theta \sum_{j=1}^{\beta} \sum_{r=1}^{\alpha} g\left(h\left(e_{r}, e_{r}\right), F P e_{j}^{*}\right)^{2} .
$$

Using Lemma 3.4 for a mixed totally geodesic warped product submanifold and the fact that $\eta\left(e_{j}\right)=0,1 \leq j \leq d_{1}-1$ for an orthonormal frame, we arrive at

$$
\|h\|^{2} \geq \csc ^{2} \theta \sum_{j=1}^{\beta} \sum_{r=1}^{\alpha}\left(P e_{j}^{*} \ln f\right)^{2} g\left(e_{r}, e_{r}\right)+\csc ^{2} \theta \cos ^{2} \theta \sum_{j=1}^{\beta} \sum_{r=1}^{\alpha}\left(e_{j}^{*} \ln f\right)^{2} g\left(e_{r}, e_{r}\right) .
$$

Hence by hypothesis, we obtain

$$
\|h\|^{2} \geq \alpha \csc ^{2} \theta \sum_{j=1}^{\beta}\left(P e_{j}^{*} \ln f\right)^{2}+\alpha \cot ^{2} \theta \sum_{j=1}^{\beta}\left(e_{j}^{*} \ln f\right)^{2} .
$$

By the property of trigonometric functions, we arrive at

$$
\|h\|^{2} \geq \alpha \csc ^{2} \theta \sum_{j=1}^{2 \beta}\left(e_{j}^{*} \ln f\right)^{2}-\alpha \sum_{j=1}^{\beta}\left(e_{j}^{*} \ln f\right)^{2} .
$$

Then by adding and subtracting the same terms $\xi \ln f$ in the above equation, we get

$$
\|h\|^{2} \geq \alpha \csc ^{2} \theta \sum_{j=1}^{2 \beta+1}\left(e_{j}^{*} \ln f\right)^{2}-\alpha \sum_{j=1}^{\beta}\left(e_{j}^{*} \ln f\right)^{2}-\alpha \csc ^{2} \theta(\xi \ln f)^{2} .
$$

$\xi \ln f=1$ [13] for a warped product submanifold of a nearly Kenmotsu manifold. Thus the above equation gives

$$
\|h\|^{2} \geq \alpha\left[\csc ^{2} \theta\left\{\left\|\nabla^{\theta} \ln f\right\|^{2}-1\right\}-\sum_{j=1}^{\beta}\left(e_{j}^{*} \ln f\right)^{2}\right] .
$$

If the equality holds, from the terms left in (4.6) and (4.7), we obtain the following conditions from the first and third terms:

$$
\|h(\mathcal{D}, \mathcal{D})\|^{2}=0, \quad g\left(h\left(\mathcal{D}^{\perp}, \mathcal{D}^{\perp}\right), \phi \mathcal{D}^{\perp}\right)=0,
$$

and

$$
g\left(h\left(\mathcal{D}^{\perp}, \mathcal{D}^{\perp}\right), v\right)=0,
$$


where $\mathcal{D}=\mathcal{D}^{\theta} \oplus \xi$, this means that $M_{\theta}$ is a totally geodesic in $\widetilde{M}$ and $h\left(\mathcal{D}^{\perp}, \mathcal{D}^{\perp}\right) \subseteq F \mathcal{D}^{\theta}$. Now from Lemma 3.4 for a mixed totally geodesic submanifold we find

$$
g(h(Z, W), F X)=-(P X \ln f) g(Z, W),
$$

for any $Z, W \in \Gamma\left(T M_{\perp}\right)$ and $X \in \Gamma\left(T M_{\theta}\right)$. The above equations imply that $M_{\perp}$ is totally umbilical in $\widetilde{M}$, so the equality holds. This completes the proof of the theorem.

\section{Competing interests}

The authors declare that they have no competing interests.

\section{Authors' contributions}

All authors contributed equally to the writing of this paper. All authors read and approved the final manuscript.

\section{Author details}

${ }^{1}$ Institute of Mathematical Sciences, Faculty of Science, University of Malaya, Kuala Lumpur, 50603, Malaysia.

${ }^{2}$ Department of Mathematics, Dokuz Eylul University, Tinaztepe Campus Buca, Izmir, 35160, Turkey.

\section{Acknowledgements}

The authors would like to express their appreciation to the referees for their comments and valuable suggestions. This work is supported by the University of Malaya research grants RG278-14AFR and RG270-13AFR.

Received: 10 April 2015 Accepted: 28 August 2015 Published online: 18 September 2015

\section{References}

1. Chen, BY: Geometry of warped product CR-submanifold in Kaehler manifolds. Monatshefte Math. 133(3), 177-195 (2001)

2. Chen, BY: Geometry of warped product submanifolds: a survey. J. Adv. Math. Stud. 6(2), 1-43 (2013)

3. Sahin, B: Warped product submanifolds of a Kaehler manifolds with slant factor. Ann. Pol. Math. 95, 207-226 (2009)

4. Khan, MA, Uddin, S, Sachdeva, R: Semi-invariant warped product submanifolds of cosymplectic manifolds. J. Inequal. Appl. 2012, Article ID 19 (2012)

5. Mustafa, A, Uddin, S, Wong, WR: Generalized inequalities on warped product submanifolds in nearly trans-Sasakian manifolds. J. Inequal. Appl. 2014, Article ID 346 (2014)

6. Uddin, S, Khan, VA: An inequality for contact CR-warped product submanifolds of cosymplectic manifolds. J. Inequal. Appl. 2012, Article ID 304 (2012)

7. Kenmotsu, K: A class of almost contact Riemannian manifolds. Tohoku Math. J. 24, 93-103 (1972)

8. Lotta, A: Slant submanifolds in contact geometry. Bull. Math. Soc. Sci. Math. Roum. 39, 183-198 (1996)

9. Cabrerizo, JL, Carriazo, A, Fernandez, LM, Fernandez, M: Slant submanifolds in Sasakian manifolds. Glasg. Math. J. 42, 125-138 (2000)

10. Carriazo, A: New Developments in Slant Submanifolds. Narosa Publishing House, New Delhi (2002)

11. Carriazo, A: Bi-slant immersions. In: Proc. ICARAMS 2000, Kharagpur, India, pp. 88-97 (2000)

12. Bishop, RL, O'Neil, B: Manifolds of negative curvature. Trans. Am. Math. Soc. 145, 1-9 (1969)

13. Uddin, S, Othman, WAM, Ozel, C: Non-existence of warped product submanifolds of nearly Kenmotsu manifolds. Bull. Iran. Math. Soc. (2014, submitted)

\section{Submit your manuscript to a SpringerOpen ${ }^{\ominus}$ journal and benefit from:}

- Convenient online submission

Rigorous peer review

- Immediate publication on acceptance

- Open access: articles freely available online

- High visibility within the field

- Retaining the copyright to your article 